\title{
Assessment of Nutritional Needs for Patients Undergoing Hemodialysis
}

Dina Gamal Hussein, Prof. Magda Abd El-Aziz Mohammed, Assist prof. Eman Saleh Shahin, Dr. Amal Baker Abo El-ata, Dr. Doaa Hamed Mohamed

Clinical Instructor, Technical Health Institute, Cairo, Medical - Surgical Nursing, Faculty of Nursing/ Ain Shams University, Medical - Surgical Nursing, Faculty of Nursing / Port Said University, Medical - Surgical Nursing, Faculty of Nursing/ Port

Said University, Nutrition, National Nutrition Institute

\begin{abstract}
Background: Chronic kidney disease (CKD) is a prevalent chronic condition and the incidence of End-Stage Renal Disease (ESRD) is expected to continue to climb in the coming decade. CKD has significant health and a lifestyle implication for those affected, including increased risk of cardiovascular disease, malnutrition and is a public health burden particularly in those patients who progress to end stage renal failure or ESRD. This study aimed to: assess nutritional needs for patients undergoing hemodialysis. A descriptive design was applied in the current study. The study was conducted at hemodialysis department at El -Hussein university hospital in Cairo and at Port Said hospitals disturbed as following, Port Said General Hospital and PortFouad General Hospital. A convenience sample of 100 patients undergoing hemodialysis was included. Three tools were used for data collection; Structured interview questionnaire, Dietary assessment questionnaire and Nutritional needs assessment questionnaire. Results of the study revealed that, more than three quarters of studied HD patients (80\%) have abnormalities of nutritional parameters and there are a statically significances between sociodemographic variables, some of nutritional needs and dietary habits whereas $(\mathrm{p}<0.05)$. Conclusion: abnormalities of nutritional parameters are over prevalent than expected by analysis of dietary food intake. The current study recommended that, dietetic counseling is very important in HD patients, as well as a reevaluation of the type of dietary protein and energy requirements.
\end{abstract}

Keywords: End Stage Renal Disease, Chronic Kidney Disease, Hemodialysis, Nutritional Needs. 


\section{INTRODUCTION}

Chronic kidney disease (CKD) constitutes a serious public health problem associated with rising incidence and prevalence, the high cost of treatment and poor outcomes. In the last decade, there has been an exponential increase in the incidence and prevalence of CKD worldwide, such that CKD has assumed epidemic proportions in both developing and developed countries (Amira \& Bello, 2015). In Egypt, the prevalence of end- stage renal disease (ESRD) is 483 per million populations and the total recorded number of ESRD patients on dialysis is 40000 (Afifi \& Moustafa, 2008).

Chronic kidney disease is usually an irreversible and progressive disease and can lead to kidney failure, also called end- stage renal disease (ESRD), over time if it is not treated. Once detected, CKD can be treated through medication and lifestyle changes to slow down the disease progression, and to prevent or delay the onset of kidney failure. However, the only treatment options for kidney failure are dialysis or a kidney transplantation (Centers for Disease Control and Prevention, 2010).

Dialysis is a treatment to filter wastes and water from the blood, allowing people with kidney failure to feel better and continue doing the things they enjoy. In hemodialysis (HD), blood goes through a filter outside body and the clean blood is returned to the body. HD is usually done at a dialysis center three times a week (National Kidney Disease Education Program, 2014).

Individuals undergoing dialysis have a significant prevalence of malnutrition, which is classified as mild, moderate, and severe (De Oliveira \& Andrade, 2012). Malnutrition is a general term that indicates a state of nutrition in which a deficiency or excess (or imbalance) of energy, protein, and other nutrients causes measurable adverse effects on tissue/body form (body shape, size, and composition) and function, and clinical outcome. The first and most important type is protein-energy wasting (PEW), which is defined as a lack in supply of sufficient energy or protein to meet the body's metabolic demands (Poulia \& Baschali, 2014).

Nutritional management is widely recognized as an integral part of the treatment for patients with CKD. Patients undergoing $\mathrm{HD}$ are at a high risk of malnutrition this significantly impacts on mortality. Therefore, in order to optimize nutritional status, it is important for those patients with end- stage kidney disease on HD are given appropriate nutritional requirements are met (De Mutsert \& Grootendorst, 2009).

Patients on maintenance hemodialysis should have a minimum protein intake of $1.1 \mathrm{~g} / \mathrm{kg}$ IBW (Ideal Body Weight) /day (Macafee\& Magee, 2011). To achieve good dialysis outcome, dialysis patients need to strictly control their diet so as to help control the waste products and fluids accumulated between dialysis treatments. It is essential for dialysis patients to have the right amount intake of protein, calories, fluids, vitamins and minerals each day. A good diet for a dialysis patient is adequate 
in protein, adequate in calories, low in potassium, low in sodium, and low in phosphorus, controlled in fluids (Cahyaningsih, 2009).

The identification and management of early chronic kidney disease are the responsibility of primary and secondary health care professionals (Thomas $\&$ Coldstream, 2006). The dialysis nurse has an important role, the not only identification and management of these patients but also in patient's education about kidney disease and lifestyle modifications. Evidence suggests that nurses can improve care by communicating effectively with patients and helping them to understand their illness and concordance with medication and treatment regimens (Burrows, 2005).

The nurse who cares for a patient with symptoms or complications resulting from dietary indiscretion must avoid harsh, judgmental, or punitive tones when communicating with him or her. Regular education with reinforcement is needed to achieve this difficult change in lifestyle (Smeltzer\&Bare, 2012).

\section{Significance of the study:}

Nutrition management is an important component in the prevention of malnutrition in patients with moderate to severe kidney disease. Renal insufficiency and chronic renal failure require nutritional treatment that includes dietary modifications in potassium, protein, and sodium intake based on the patient's nutrition status. These changes are incorporated to lessen uremic symptoms and slow the decline in kidney function (Leslie, 2011). It is the role of the dialysis nurse to identify patients at risk from malnutrition and do a nursing intervention about their condition and make an appropriate referral to the dietitian (Cahyaningsih, 2009).

The nutrition has a vital role in decreasing mortality and morbidity in patients undergoing hemodialysis. The information available about nutritional needs and status of dialysis patients in developing countries; including Egypt, is very little compared to the USA and Europe. Therefore, this study was carried out to assess nutritional needs of patients undergoing hemodialysis.

\section{AIM OF THIS STUDY:}

Assess nutritional needs for patients undergoing hemodialysis.

\section{Research Question:}

To achieve the aim of this study, the following research questions are formulated:

A- What are the nutritional needs for patients undergoing hemodialysis?

B- What are the Sociodemographic factors affecting the nutritional needs for patients undergoing hemodialysis? 


\section{SUBJECTS AND METHODS:}

\section{Subjects}

\section{Research design:}

A descriptive design was used in this study to fulfill the aim of the study and answer the research questions.

\section{Setting:}

This study was carried out in hemodialysis units at El -Hussein university hospital in Cairo and at Port Said city governmental hospitals disturbed as following: - (Port Said general hospital and Port-Fouad general hospital).

\section{Subjects}

A convenience sample of (100) patients in hemodialysis units during the six months from the beginning of February, 2014, to the end of July, 2014 were included in the study.

Inclusion criteria were: Patients on hemodialysis for at least 6 months; both sexes; age 18 years and older and patients suffer from chronic disease only hypertension and diabetes.

Exclusion criteria were: The patients suffer from any chronic disease as chest diseases, hepatitis, and cardiac diseases.

Tools of data collection: Three tools were used in this study to collect the necessary data.

Tool I: Structured Interviewing Questionnaire: was developed by the researcher after reviewing and utilizing the most recent and relevant literature, it was consisted of four parts:

Part 1: It was concerned with socio-demographic characteristics of patients under study such as (sex, age, marital status, occupation..... etc.

Part 2: Anthropometric measurements questionnaire, it was used to assess nutritional status of HD patients, such as (Height, IDWG (Interdialytic weight gain) and weight before dialysis, BMI (body mass index) ... etc.

Part 3: Dialysis history and laboratory investigations after sessions, it was used to assess health status of HD patients and kidney profile for each hemodialysis patient, such as (number of session (T/wk), duration of dialysis/session, start of dialysis (years), other diseases, other associated medical problems, findings of investigations...etc.

Part 4: Dietary habits, it was used to assess dietary habits for each hemodialysis patient such as (loss of appetite, method of cooking, leaching of potassium $(\mathrm{K}+)$ ...etc.

Tool II: Questionnaire Sheet to Assess Dietary Intake: was developed by the researcher. It was consisted of two parts:

Part 1: Food Frequency Questionnaire, It was used to find out a frequency of the most common consumed food per week such as (Grains \&starch, tubers, vegetables, fruits etc. 
Part 2: 24 Recall Assessment Questionnaire, it is a three days dietary record was used to assess consumption of food and beverages such as (meals, foods, drinks names...... etc. The responses were recorded on the questionnaires.

The portion sizes of foods consumed by each patient were converted into percent CHO, fats proteins and kcal and analysis by food composition 2006.

Tool III: Nutritional needs assessment Questionnaire: it is a guidelines was developed by Rucker 2010; Fouque et al. 2007 and modified by the researcher, which included (macronutrients \& fiber, minerals \& water, some vitamins dietary intake guidelines)>

\section{Methods}

\section{Ethical Considerations:}

An official litter was directed from the dean of Faculty of Nursing, Port - Said University to the hospitals managers. Written permission to conduct the study was obtained from the director of each setting and the head of hemodialysis units of the selected hospitals after explaining the purpose of the study to gain their cooperation during the period of the study. Verbal consents were obtained from each participant (patients) to be included in the study after explaining clarification of the nature and purpose of the study. The researcher was emphasized that, the participations were absolutely voluntary and each patients have the right to withdraw from the study at any time without explaining any reasons, as well as confidentiality was assured.

\section{Content Validity and Reliability:}

Content validity was tested by seven experts from Faculty of Nursing in the field of Medical - Surgical Nursing and physicians from faculty of medicine and dietitian from national nutrition institute. The questionnaire was modified according to the experts' comments and recommendations and the tools tested for reliability. The internal consistency of tools has been tested using Cronbach's alpha coefficient. Cronbach's alpha for dietary habits questionnaire, food frequency questionnaire, 24 recall assessment questionnaire and nutritional needs assessment questionnaire were $(0.79,0.86,0.83, \& 0.88)$ respectively.

\section{Pilot Study:}

A pilot study was done to assess the feasibility and applicability of tools and to estimate the proper time required for answering the questionnaire. It was conducted on10.0 \% of patients from previously mentioned selected settings. The patients included in the pilot study were excluded from the main sample.

\section{Field of work:}

An official written permission to conduct the study was obtained from the director of each setting and the head of hemodialysis units of the selected hospitals, and verbal consents were obtained from each participant (patients) to be included in the study after explaining clarification of the nature and purpose of the study. A verbal consent 
was obtained from each patient and wrote his name on the questionnaire sheet after the researcher introducing himself to the patient and explaining the purpose of the study at the beginning of the interview. The patients were assured that, all information will be confidential and will be used only for the purpose of the study.

Data were collected over a period of six months from the beginning of February, 2014, to the end of July, 2014. Data were collected for three days per week for each hospital (Saturday, Sunday, and Monday in Cairo and Tuesday, Wednesday, and Thursday in Port Said) at morning shift from 8:00 am to 2:00 pm and afternoon shifts from 2:00 pm to 8:00 $\mathrm{pm}$.

Data were collected using written questionnaire sheet for each patient that was interviewed individually to fill in the questionnaire sheet by the researcher which was read the questionnaire to the patients and as they answered the researcher filled in the questionnaire. The all-time needed for the application of the tool was lasted approximately about 40-45 minutes. First tool was structured interviewing questionnaire; it lasts about 15 minutes to fill in it, while the second tool was assess dietary intake it lasts about 25-30 minutes to fill in it.

\section{Statistical Data Analysis:}

Data were extracted from the interview questionnaire and computerized in Microsoft Excel 2007. Data analyzed was done using Chi-Square test computer software package, while statistical analysis was done using the statistical package for social sciences (SPSS) version 18.0. Data were presented using descriptive statistics in the form of frequencies and percentages for qualitative variables, and means and standard deviations for quantitative variables. A significant level value was considered when $\mathrm{p}$ value $\leq 0.05$.

\section{Study limitations:}

The length of time to obtain approval of the hospitals in which the sample collect. The dietary history method includes under-reporting and inaccurate recall, which may lead to inaccurate estimates of nutrient consumption.

\section{RESULTS:}

The Results showed that, more than one third of studied HD patients (35\%) were at the age group 30 to less than 40 years, with the mean age of patients under study was (43.1 \pm 8.4$)$. Regarding gender; half of them $(50 \%)$ were males and $(50 \%)$ were females in the current study. As regard to level of education, half of studied HD patients $\quad(50 \%)$ were at intermediate school level, while $(13 \%)$ were Illiterate. Concerning occupation, more than one -third of studied HD patients $(39 \%)$ were house wife, while the minority of them $(5 \%)$ was other work. In relation to marital status, most of studied HD patients $(78 \%)$ were married, while the minority of them (6\%) was divorced. Regarding to monthly income, the majority of patients $(97 \%)$ were enough according to self-report, and the minority of them (3\%) were not enough 
Table (1): As regard to anthropometric measurements among studied hemodialysis patients indicated that, more than half of studied HD patients (54\%) have a BMI 25$29.9 \mathrm{~kg} / \mathrm{m}^{2}$ being overweight (Fig1). Regarding IDWG most of studied HD patients (85\%) have IDWG 2-5\%. Concerning weight change in 2 weeks ago, less than two third of them (62\%) have weight change in 2 weeks ago more than $2 \%$.In relation to weight change in 6 month ago, most of studied HD patients (79\%) have weight change in 6 month ago less than 5\%. As regard to urinary output (UOP), more than half of studied HD patients (57\%) have UOP amount with mean of $(34 \pm 37.08)$ in Port Said and Cairo.

Table (2): Concerning dialysis history and associated diseases among studied hemodialysis patients indicated that most of studied HD patients $(69 \%)$ have been on dialysis more than 2 years and less than 5 years, In relation to other associated medical problems with CKD, more than one- third of studied HD patients $(36 \%)$ suffering from hypertension and more than half (51\%) of them don't suffer from other diseases with CKD, Regarding family history of CKD, most of the patients of studied HD patients (76\%) have no a family history of CKD in Port Said and Cairo.

(Table 3): Regarding laboratory investigations among studied hemodialysis patients indicated that, the mean value of BUN was $119.68 \pm 37.47$. The mean value of albumin was $3.75 \pm 0.581$. The mean value of hemoglobin was $10.341 \pm 1.95$. The mean value of phosphorus was $5.894 \pm 1.498$. The mean value of sodium was $154.03 \pm 19.26$. The mean value of cholesterol was $179.3 \pm 45.85$.

(Table 4): When analyzing the dietary habits among studied hemodialysis patients indicated that, most of studied HD patients ( $82 \%)$ have normal appetite. In relation to method of cooking, more than two- fifths of studied HD patients (47\%) used foundry and sautéed method in cooking. As regard to leaching of potassium, most of patients $(86 \%)$ did not used the ways to get rid of potassium. Concerning eating fast foods, more than half of patients (58\%) eat outdoor and most of HD patients (81\%) eat pickles. There was a statistically significance relation in eating fast foods between Port Said and Cairo whereas $\left(\mathrm{P}=0.013^{*}\right)$.

(Table 5): In relation to adequacy of macronutrients and fiber among studied hemodialysis patients indicated that, the majority of patients (98\%) are taking less than $35 \mathrm{kcal} / \mathrm{kg} / \mathrm{day}$. While, more than two- fifths of studied HD patients (49\%) are taking more than or equal $1.2 \mathrm{~g} / \mathrm{kg} / \mathrm{d}$ DPI and most of the ingested protein $(98 \%)$ of the patients comes from high biological value less than or equal $50 \%$. In addition, most of studied HD patients $(80 \%)$ are taking more than or equal $7 \%$ saturated fats. However, the majority of patients (99\%) are taking less than $20 \mathrm{~g} / \mathrm{d}$ dietary fiber. There is no a statistically significance relation in dietary intake of macronutrients and fiber between Port Said and Cairo $(\mathrm{p}<0.05)$.

(Table 6): Concerning dietary intake of minerals and water among studied hemodialysis patients revealed that, most of studied HD patients $(87 \%)$ are taking 
more than $2000 \mathrm{mg} / \mathrm{d}$ dietary sodium. Also, most of patients (89\%) are taking more than $1000 \mathrm{~mL} / \mathrm{d}$ of fluid intake. In addition, most of patients $(82 \%)$ are taking less than $40 \mathrm{mEq} / \mathrm{IBW} / \mathrm{d}$ dietary potassium. While, more than half of studied HD patients (57\%) are taking more than $16 \mathrm{mg} / \mathrm{IBW} / \mathrm{d}$ dietary phosphorus intake. There was a statistically significance relation in dietary intake of fluid between Port Said and Cairo whereas $\left(\mathrm{P}=0.025^{*}\right)$.

(Table 7): Regarding to dietary intake of some vitamins among studied hemodialysis patients indicated that, the majority of patients have some of vitamins malnutrition as (A, B1, B2, C) which the intake below the recommended level. There is no statistically significance relation in dietary intake of some vitamins between Port Said and Cairo $(\mathrm{p}<0.05)$

(Table 8): In relation to sociodemographic data, the current study was found a statistical significance between sex and potassium $\left(\mathrm{P}=.009^{*}\right)$. In addition, there was a statistical significance relation between sex and eating fast foods in Cairo ( $\mathrm{P}=.031^{*}$ ). The current study was elicited that, there was a statistically significance between age and sodium intake in Port Said $\left(\mathrm{P}=.006^{*}\right)$. Also, there was a statistically significance between age and dietary habits (eating pickles and fast food) in Cairo ( $\left.\mathrm{P}=.011^{*}\right)$ $(\mathrm{P}=.049 *)$, respectively. In addition, There was a statistically significance between education and protein intake in Port Said which intermediate school HD patients showed higher protein intake more than $1.2 \mathrm{~g} / \mathrm{kg} / \mathrm{d}\left(\mathrm{P}=.031^{*}\right)$ compared to intermediate school HD patients in Cairo showed lower protein intake less than $1 \mathrm{~g} / \mathrm{kg}$ $\left(\mathrm{P}=.025^{*}\right)$. However, There was a statistically significance between education and some vitamins intake as $\mathrm{A}, \mathrm{B} 1$ and $\mathrm{B} 2\left(\mathrm{P}=.033^{*}\right)\left(\mathrm{P}=.008^{*}\right)\left(\mathrm{P}=.025^{*}\right)$ respectively, which vitamins intake lower than recommended level in both groups. Also, there was a statistically significance relation between marital status and protein intake in Cairo $\left(\mathrm{P}=.013^{*}\right)$.

Figure (1): Body mass index (BMI) among studied hemodialysis patients $(n=100)$

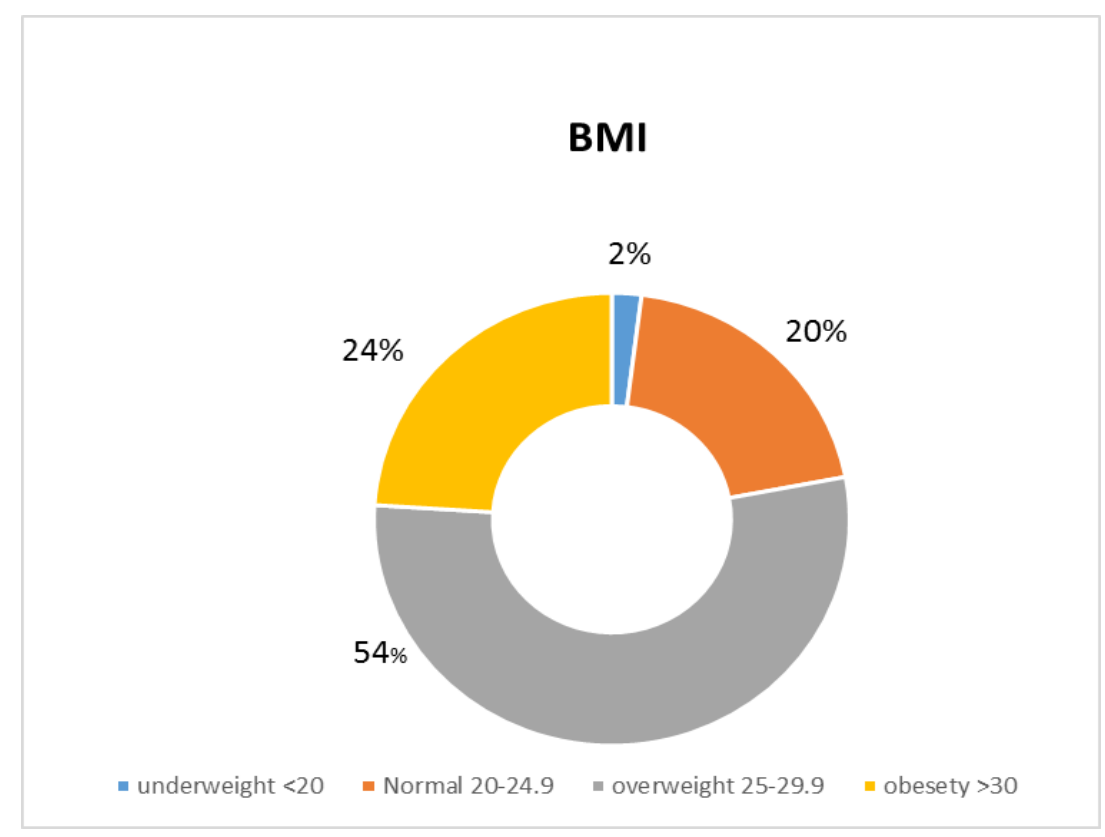


Table (1): Socio-demographic characteristics of studied hemodialysis patients $(n=100)$

\begin{tabular}{|c|c|c|c|c|c|c|c|}
\hline \multirow{2}{*}{\multicolumn{2}{|c|}{ Personal characteristics }} & \multicolumn{2}{|c|}{$\begin{array}{l}\text { Port Said } \\
\mathbf{n}=\mathbf{5 0}\end{array}$} & \multicolumn{2}{|c|}{$\begin{array}{l}\text { Cairo } \\
\mathbf{n}=\mathbf{5 0}\end{array}$} & \multicolumn{2}{|c|}{$\begin{array}{l}\text { Total } \\
\mathrm{n}=100\end{array}$} \\
\hline & & No & $\%$ & No & $\%$ & $\overline{\text { No }}$ & $\%$ \\
\hline \multirow{2}{*}{ Sex } & Female & 25 & 50 & 25 & 50 & 50 & 50 \\
\hline & Male & 25 & 50 & 25 & 50 & 50 & 50 \\
\hline \multirow{5}{*}{ Age } & $20<30$ years & 4 & 8 & 1 & 2 & 5 & 5 \\
\hline & $30<40$ years & 15 & 30 & 20 & 40 & 35 & 35 \\
\hline & $40<50$ years & 16 & 32 & 15 & 30 & 31 & 31 \\
\hline & $50-60$ years & 15 & 30 & 14 & 28 & 29 & 29 \\
\hline & \multicolumn{3}{|l|}{ Mean \pm SD } & \multicolumn{4}{|c|}{$43.1 \pm 8.4$} \\
\hline \multirow{4}{*}{ Education } & Illiterate & 3 & 6 & 10 & 20 & 13 & 13 \\
\hline & Read and write & 4 & 8 & 13 & 26 & 17 & 17 \\
\hline & $\begin{array}{l}\text { Intermediate } \\
\text { School }\end{array}$ & 30 & 60 & 20 & 40 & 50 & 50 \\
\hline & University Degree & 13 & 26 & 7 & 14 & 20 & 20 \\
\hline \multirow{5}{*}{ Occupation } & Officer (Employed) & 21 & 42 & 7 & 14 & 28 & 28 \\
\hline & Worker & 1 & 2 & 5 & 10 & 6 & 6 \\
\hline & Housewife & 15 & 30 & 24 & 48 & 39 & 39 \\
\hline & $\begin{array}{l}\text { None } \\
\text { (Unemployed) }\end{array}$ & 10 & 20 & 12 & 24 & 22 & 22 \\
\hline & Other & 3 & 6 & 2 & 4 & 5 & 5 \\
\hline \multirow{4}{*}{ Marital Status } & Single & 4 & 8 & 4 & 8 & 8 & 8 \\
\hline & Married & 40 & 80 & 38 & 76 & 78 & 78 \\
\hline & Divorced & 1 & 2 & 5 & 10 & 6 & 6 \\
\hline & Widower & 5 & 10 & 3 & 6 & 8 & 8 \\
\hline \multirow{2}{*}{ Income } & Enough & 47 & 94 & 50 & 100 & 97 & 97 \\
\hline & Is not enough & 3 & 6 & 0 & 0 & 3 & 3 \\
\hline
\end{tabular}

Table (2): Anthropometric measurements among studied hemodialysis patients $(n=100)$

\begin{tabular}{|c|c|c|c|c|c|c|c|}
\hline \multicolumn{2}{|l|}{ Anthropometric Measurements } & \multicolumn{2}{|c|}{$\begin{array}{l}\text { Port Said } \\
\mathrm{n}=\mathbf{5 0}\end{array}$} & \multicolumn{2}{|c|}{$\begin{array}{l}\text { Cairo } \\
\mathbf{n}=\mathbf{5 0}\end{array}$} & \multicolumn{2}{|c|}{$\begin{array}{l}\text { Total } \\
\mathbf{n}=100\end{array}$} \\
\hline & & No & $\%$ & No & $\%$ & No & $\%$ \\
\hline \multirow{3}{*}{$\begin{array}{l}\begin{array}{l}\text { Intradialytic } \\
\text { (IDWG) } \%\end{array} \\
\text { Weight }\end{array}$} & $<2 \%$ & 0 & 0 & 1 & 2 & 1 & 1 \\
\hline & $2-5 \%$ & 48 & 96 & 37 & 74 & 85 & 85 \\
\hline & $>5 \%$ & 2 & 4 & 12 & 24 & 14 & 14 \\
\hline \multirow{3}{*}{$\begin{array}{l}\text { Weight }(\mathrm{Wt}) \text { change } \\
2 \text { weeks ago }\end{array}$} & $<1 \%$ & 16 & 32 & 5 & 10 & 21 & 21 \\
\hline & $1: 2 \%$ & 12 & 24 & 5 & 10 & 17 & 17 \\
\hline & $>2 \%$ & 22 & 44 & 40 & 80 & 62 & 62 \\
\hline \multirow{3}{*}{$\begin{array}{l}\text { Weight }(\mathrm{Wt}) \text { change } \\
6 \text { month ago }\end{array}$} & $<5 \%$ & 40 & 80 & 39 & 78 & 79 & 79 \\
\hline & $\begin{array}{l}5 \\
10 \%\end{array}$ & 10 & 20 & 9 & 18 & 19 & 19 \\
\hline & $>10 \%$ & 0 & 0 & 2 & 4 & 2 & 2 \\
\hline \multirow{2}{*}{$\begin{array}{l}\text { Urinary Out Put } \\
\text { (UOP) }\end{array}$} & Yes & 33 & 66 & 24 & 48 & 57 & 57 \\
\hline & No & 17 & 34 & 26 & 52 & 43 & 43 \\
\hline $\begin{array}{l}\begin{array}{l}\text { Urinary Out Put } \\
\text { amount } / 24 \text { hour }\end{array} \\
\text { (UOP) }\end{array}$ & \multicolumn{3}{|c|}{ Mean \pm SD } & \multicolumn{4}{|c|}{$34 \pm 37.08$} \\
\hline
\end{tabular}


Table (3): Dialysis history and associated diseases among studied hemodialysis patients $(\mathrm{n}=100)$

\begin{tabular}{|c|c|c|c|c|c|c|c|}
\hline \multirow{2}{*}{\multicolumn{2}{|c|}{ Dialysis history and associated diseases }} & \multicolumn{2}{|c|}{$\begin{array}{c}\text { Port Said } \\
\mathbf{n}=\mathbf{5 0}\end{array}$} & \multicolumn{2}{|c|}{$\begin{array}{l}\text { Cairo } \\
\mathbf{n}=\mathbf{5 0}\end{array}$} & \multicolumn{2}{|c|}{$\begin{array}{r}\text { Total } \\
n=100\end{array}$} \\
\hline & & No & $\%$ & No & $\%$ & No & $\%$ \\
\hline \multirow{3}{*}{$\begin{array}{l}\text { Number of sessions } \\
\text { (T/wk) }\end{array}$} & $<3$ & 0 & 0 & 0 & 0 & 0 & 0 \\
\hline & 3 & 50 & 100 & 50 & 100 & 100 & 100 \\
\hline & $>3$ & 0 & 0 & 0 & 0 & 0 & 0 \\
\hline \multirow{3}{*}{ Duration of HD/session } & 3 & 0 & 0 & 0 & 0 & 0 & 0 \\
\hline & 3.5 & 0 & 0 & 0 & 0 & 0 & 0 \\
\hline & 4 & 50 & 100 & 50 & 100 & 100 & 100 \\
\hline \multirow{4}{*}{ Start of dialysis (years) } & $<2$ years & 15 & 30 & 6 & 12 & 21 & 21 \\
\hline & $2-5$ years & 30 & 60 & 39 & 78 & 69 & 69 \\
\hline & $6-10$ years & 4 & 8 & 2 & 4 & 6 & 6 \\
\hline & $>10$ years & 1 & 2 & 3 & 6 & 4 & 4 \\
\hline \multirow{5}{*}{$\begin{array}{l}\text { Other associated medical } \\
\text { problems }\end{array}$} & DM & 1 & 4.2 & 0 & 0 & 1 & 1 \\
\hline & HTP & 15 & 62.5 & 21 & 84 & 36 & 36 \\
\hline & HTP+DM & 8 & 33.3 & 4 & 16 & 12 & 12 \\
\hline & Total & 24 & 100 & 25 & 100 & 49 & 49 \\
\hline & & 26 & 52 & 25 & 50 & 51 & 51 \\
\hline \multirow{2}{*}{ Family history of CKD } & \begin{tabular}{|l|} 
Yes \\
\end{tabular} & 16 & 32 & 8 & 16 & 24 & 24 \\
\hline & \begin{tabular}{|l} 
No \\
\end{tabular} & 34 & 68 & 42 & 84 & 76 & 76 \\
\hline
\end{tabular}

Table (4): Laboratory investigations after sessions among studied hemodialysis patients $(\mathrm{n}=100)$

\begin{tabular}{|c|c|c|c|c|c|c|c|c|c|}
\hline \multicolumn{3}{|c|}{ Laboratory investigations } & \multicolumn{2}{|c|}{$\begin{array}{l}\text { Port Said } \\
\mathbf{n}=\mathbf{5 0}\end{array}$} & \multicolumn{2}{|c|}{$\begin{array}{l}\text { Cairo } \\
\mathbf{n}=\mathbf{5 0}\end{array}$} & \multicolumn{2}{|c|}{$\begin{array}{l}\text { Total } \\
\mathbf{n}=\mathbf{1 0 0}\end{array}$} & \multirow{2}{*}{ Mean \pm SD } \\
\hline & & & No & $\%$ & No & $\%$ & No & $\%$ & \\
\hline \multirow{3}{*}{\multicolumn{2}{|c|}{$\begin{array}{l}\text { Blood urea nitrogen } \\
\text { BUN) } \mathrm{mg} / \mathrm{dl}(\end{array}$}} & $<40$ & 0 & 0 & 1 & 2 & 1 & 1 & \multirow{3}{*}{$119.68 \pm 37.47$} \\
\hline & & $40-60$ & 6 & 12 & 0 & 0 & 6 & 6 & \\
\hline & & $>60$ & 44 & 88 & 49 & 98 & 93 & 93 & \\
\hline \multirow{2}{*}{\multicolumn{2}{|c|}{$\begin{array}{l}\text { Albumin } \\
\mathrm{g} / \mathrm{dl}\end{array}$}} & $<4$ & 25 & 50 & 30 & 60 & 55 & 55 & \multirow[t]{2}{*}{$3.75 \pm 0.581$} \\
\hline & & $\geq 4$ & 25 & 50 & 20 & 40 & 45 & 45 & \\
\hline \multirow{6}{*}{$\begin{array}{l}\text { Hemoglobin (HB) } \\
\mathrm{g} / \mathrm{dl}\end{array}$} & \multirow{3}{*}{ 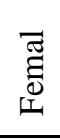 } & $<10$ & 13 & 13 & 8 & 8 & 21 & 21 & \multirow{6}{*}{$10.34 \pm 1.95$} \\
\hline & & $10-12$ & 10 & 10 & 13 & 13 & 23 & 23 & \\
\hline & & $>12$ & 2 & 2 & 4 & 4 & 6 & 6 & \\
\hline & \multirow{3}{*}{$\frac{0}{\pi}$} & $<10$ & 14 & 14 & 6 & 6 & 20 & 20 & \\
\hline & & $10-12$ & 10 & 10 & 10 & 10 & 20 & 20 & \\
\hline & & $>12$ & 1 & 1 & 9 & 9 & 10 & 10 & \\
\hline \multirow{3}{*}{\multicolumn{2}{|c|}{$\begin{array}{l}\text { Potassium (K) } \\
\mathrm{mg} / \mathrm{dl}\end{array}$}} & $<3.5$ & 0 & 0 & 0 & 0 & 0 & 0 & \multirow[t]{3}{*}{$5.022 \pm 0.898$} \\
\hline & & $3.5-5.3$ & 39 & 78 & 38 & 76 & 77 & 77 & \\
\hline & & $>5.3$ & 11 & 22 & 12 & 24 & 23 & 23 & \\
\hline \multirow{3}{*}{\multicolumn{2}{|c|}{ Phosphorus (Ph)mg/dl }} & $<3.5$ & 0 & 0 & 4 & 8 & 4 & 4 & \multirow[t]{3}{*}{$5.89 \pm 1.498$} \\
\hline & & $3.5-5.5$ & 20 & 40 & 18 & 36 & 38 & 38 & \\
\hline & & $>5.5$ & 30 & 60 & 28 & 56 & 58 & 58 & \\
\hline \multirow{3}{*}{\multicolumn{2}{|c|}{$\begin{array}{l}\text { Calcium }(\mathrm{Ca}) \\
\mathrm{mg} / \mathrm{dl}\end{array}$}} & $<8.4$ & 8 & 16 & 6 & 12 & 14 & 14 & \multirow[t]{3}{*}{$9.63 \pm 1.835$} \\
\hline & & $8.4-10.2$ & 31 & 62 & 33 & 66 & 64 & 64 & \\
\hline & & $>10.2$ & 11 & 22 & 11 & 22 & 22 & 22 & \\
\hline \multirow{3}{*}{\multicolumn{2}{|c|}{$\begin{array}{l}\text { Sodium (Na) } \\
\mathrm{mg} / \mathrm{dl}\end{array}$}} & $<135$ & 8 & 16 & 4 & 8 & 12 & 12 & \multirow[t]{3}{*}{$154.03 \pm 19.26$} \\
\hline & & $135-145$ & 9 & 18 & 12 & 24 & 21 & 21 & \\
\hline & & $>145$ & 33 & 66 & 34 & 68 & 67 & 67 & \\
\hline \multirow{2}{*}{\multicolumn{2}{|c|}{$\begin{array}{l}\text { Cholesterol } \\
(\mathrm{Chol}) \mathrm{mg} / \mathrm{dl}\end{array}$}} & $<200$ & 29 & 58 & 32 & 64 & 61 & 61 & \multirow[t]{2}{*}{$179.3 \pm 45.85$} \\
\hline & & $\geq 200$ & 21 & 42 & 18 & 36 & 39 & 39 & \\
\hline
\end{tabular}


Table (5): Dietary habits among studied hemodialysis patients $(\mathrm{n}=100)$

\begin{tabular}{|c|c|c|c|c|c|c|c|c|}
\hline \multirow{2}{*}{\multicolumn{2}{|c|}{ Dietary habits }} & \multicolumn{2}{|c|}{$\begin{array}{l}\text { Port Said } \\
n=50\end{array}$} & \multicolumn{2}{|c|}{$\begin{array}{l}\text { Cairo } \\
\mathrm{n}=50\end{array}$} & \multicolumn{2}{|c|}{$\begin{array}{l}\text { Total } \\
n=100\end{array}$} & \multirow[t]{2}{*}{ Significance } \\
\hline & & No & $\%$ & No & $\%$ & No & $\%$ & \\
\hline \multirow{2}{*}{ Loss of appetite } & Yes & 5 & 10 & 13 & 26 & 18 & 18 & \multirow{2}{*}{$\begin{array}{l}\mathrm{P}=.066 \\
\mathrm{X}^{2}=3.320\end{array}$} \\
\hline & No & 45 & 90 & 37 & 74 & 82 & 82 & \\
\hline \multirow{5}{*}{ Method Of Cooking } & Foundry & 11 & 22 & 12 & 24 & 23 & 23 & \multirow{5}{*}{$\begin{array}{l}\mathrm{P}=.513 \\
\mathrm{X}^{2}=3.272\end{array}$} \\
\hline & $\mathrm{Ne} \times \mathrm{Ne}$ & 1 & 2 & 0 & 0 & 1 & 1 & \\
\hline & $\begin{array}{ll}\begin{array}{l}\text { Sautéed } \\
\text { poached }\end{array} & \text { or } \\
\end{array}$ & 13 & 26 & 14 & 28 & 27 & 27 & \\
\hline & $\begin{array}{l}\text { Foundry+ } \\
\text { Sautéed }\end{array}$ & 25 & 50 & 22 & 44 & 47 & 47 & \\
\hline & $\begin{array}{l}\text { Sautéed }+\mathrm{Ne} x \\
\mathrm{Ne}\end{array}$ & 0 & 0 & 2 & 4 & 2 & 2 & \\
\hline \multirow{2}{*}{$\begin{array}{ll}\text { Leaching } & \text { of } \\
\text { potassium }(\mathrm{K}+) & \end{array}$} & Yes & 10 & 20 & 4 & 8 & 14 & 14 & \multirow{2}{*}{$\begin{array}{l}\mathrm{P}=.084 \\
\mathrm{X}^{2}=2.990\end{array}$} \\
\hline & No & 40 & 80 & 46 & 92 & 86 & 86 & \\
\hline \multirow{2}{*}{ Eating fast foods } & Yes & 23 & 46 & 35 & 70 & 58 & 58 & \multirow{2}{*}{$\begin{array}{l}P=.013 * \\
X^{2}=5.911\end{array}$} \\
\hline & No & 27 & 54 & 15 & 30 & 42 & 42 & \\
\hline \multirow{2}{*}{ Eating pickles } & Yes & 37 & 74 & 44 & 88 & 81 & 81 & \multirow{2}{*}{$\begin{array}{l}\mathrm{P}=.062 \\
\mathrm{X}^{2}=3.184\end{array}$} \\
\hline & No & 13 & 26 & 6 & 12 & 19 & 19 & \\
\hline
\end{tabular}

$X^{2}$ : Chi-Square test

*significant at $P \leq 0.05, \mathrm{P}=.013 *$

Table (6): Dietary adequacy of macronutrients and fiber among studied hemodialysis patients $(n=100)$

\begin{tabular}{|c|c|c|c|c|c|c|c|c|c|}
\hline \multirow{2}{*}{$\begin{array}{l}\text { Macronutrients and } \\
\text { Fiber }\end{array}$} & \multirow{2}{*}{$\begin{array}{l}\text { Normal } \\
\text { range }\end{array}$} & \multirow{2}{*}{$\begin{array}{l}\text { Patient } \\
\text { result }\end{array}$} & \multicolumn{2}{|c|}{$\begin{array}{l}\text { Port Said } \\
\mathbf{n}=\mathbf{5 0}\end{array}$} & \multicolumn{2}{|c|}{$\begin{array}{l}\text { Cairo } \\
\mathbf{n}=\mathbf{5 0}\end{array}$} & \multicolumn{2}{|c|}{$\begin{array}{l}\text { Total } \\
\mathbf{n}=\mathbf{1 0 0}\end{array}$} & \multirow{2}{*}{ Significance } \\
\hline & & & No & $\%$ & No & $\%$ & No & $\%$ & \\
\hline \multirow{2}{*}{$\begin{array}{l}\text { Dietary Energy Intake } \\
\text { (DEI) Kcal/kg/day }\end{array}$} & \multirow{2}{*}{$\begin{array}{l}35 \\
\mathrm{kcal} / \mathrm{kg} / \mathrm{d}\end{array}$} & $<35$ & 49 & 98 & 49 & 98 & 98 & 98 & \multirow{2}{*}{$\begin{array}{l}\mathrm{P}=.753 \\
\mathrm{X}^{2}=.000\end{array}$} \\
\hline & & $\geq 35$ & 1 & 2 & 1 & 2 & 2 & 2 & \\
\hline \multirow{3}{*}{$\begin{array}{l}\text { Dietary Protein Intake } \\
\text { (DPI) g/kg/day }\end{array}$} & \multirow{3}{*}{$\begin{array}{l}1-1.2 \\
\mathrm{~g} / \mathrm{kg} / \mathrm{d}\end{array}$} & $<1$ & 13 & 26 & 20 & 40 & 33 & 33 & \multirow{3}{*}{$\begin{array}{l}\mathrm{P}=.186 \\
\mathrm{X}^{2}=3.360\end{array}$} \\
\hline & & $1-1.2$ & 8 & 16 & 10 & 20 & 18 & 18 & \\
\hline & & $>1.2$ & 29 & 58 & 20 & 40 & 49 & 49 & \\
\hline \multirow{2}{*}{$\begin{array}{l}\text { Carbohydrate (CHO } \\
\text { \%)of total calories }\end{array}$} & \multirow[t]{2}{*}{$<70 \%$} & $<70 \%$ & 50 & 100 & 50 & 100 & 100 & 100 & \multirow{2}{*}{ (a) } \\
\hline & & $\geq 70 \%$ & 0 & 0 & 0 & 0 & 0 & 0 & \\
\hline \multirow{2}{*}{$\begin{array}{l}\text { High Biological Value } \\
\text { (HBV \%) }\end{array}$} & \multirow{2}{*}{$\begin{array}{ll}\text { at } & \text { least } \\
50 \% & \end{array}$} & $<50 \%$ & 48 & 96 & 50 & 100 & 98 & 98 & \multirow{2}{*}{$\begin{array}{l}\mathrm{P}=.153 \\
\mathrm{X}^{2}=3.112\end{array}$} \\
\hline & & $\geq 50 \%$ & 2 & 4 & 0 & 0 & 2 & 2 & \\
\hline \multirow{2}{*}{$\begin{array}{l}\text { Dietary Fat Intake } \\
(\text { DFI\%) of total } \\
\text { calories }\end{array}$} & \multirow[t]{2}{*}{$<30 \%$} & $<30 \%$ & 40 & 80 & 38 & 76 & 78 & 78 & \multirow{2}{*}{$\begin{array}{l}\mathrm{P}=.629 \\
\mathrm{X}^{2}=.233\end{array}$} \\
\hline & & $\geq 30 \%$ & 10 & 20 & 12 & 24 & 22 & 22 & \\
\hline \multirow{2}{*}{$\begin{array}{lll}\text { Saturated } & \text { fatty } & \text { acid } \\
\text { (SFAc) \% } & & \\
\end{array}$} & \multirow{2}{*}{$\geq 7 \%$} & $<7 \%$ & 10 & 20 & 10 & 20 & 20 & 20 & \multirow{2}{*}{$\begin{array}{l}\mathrm{P}=.598 \\
\mathrm{X}^{2}=3.245\end{array}$} \\
\hline & & $\geq 7 \%$ & 40 & 80 & 40 & 80 & 80 & 80 & \\
\hline \multirow{2}{*}{$\begin{array}{l}\text { Cholesterol (Chol) } \\
\text { g/d }\end{array}$} & \multirow{2}{*}{$<200 \mathrm{~g} / \mathrm{d}$} & $<200$ & 38 & 76 & 40 & 80 & 78 & 78 & \multirow{2}{*}{$\begin{array}{l}\mathrm{P}=.629 \\
\mathrm{X}^{2}=.324\end{array}$} \\
\hline & & $\geq 200$ & 12 & 24 & 10 & 20 & 22 & 22 & \\
\hline \multirow{2}{*}{$\begin{array}{l}\text { Fiber } \\
\text { g/d }\end{array}$} & \multirow[t]{2}{*}{$\geq 20 \mathrm{~g} / \mathrm{d}$} & $<20$ & 50 & 100 & 49 & 98 & 99 & 99 & \multirow{2}{*}{$\begin{array}{l}\mathrm{P}=.315 \\
\mathrm{X}^{2}=1.010\end{array}$} \\
\hline & & $\geq 20$ & 0 & 0 & 1 & 2 & 1 & 1 & \\
\hline
\end{tabular}


Table (7): dietary intake of minerals and fluid among studied hemodialysis patients $(\mathrm{n}=100)$

\begin{tabular}{|c|c|c|c|c|c|c|c|c|c|}
\hline \multirow[t]{2}{*}{ Minerals and Fluid } & \multirow[t]{2}{*}{ Normal range } & \multirow{2}{*}{$\begin{array}{l}\text { Patient } \\
\text { result }\end{array}$} & \multicolumn{2}{|c|}{$\begin{array}{l}\text { Port Said } \\
\mathrm{n}=50\end{array}$} & \multicolumn{2}{|c|}{$\begin{array}{l}\text { Cairo } \\
\mathbf{n}=50\end{array}$} & \multicolumn{2}{|c|}{$\begin{array}{l}\text { Total } \\
\text { n=100 }\end{array}$} & \multirow[t]{2}{*}{ Significance } \\
\hline & & & No & $\%$ & No & $\%$ & No & $\%$ & \\
\hline \multirow{3}{*}{$\begin{array}{l}\text { Sodium } \\
\text { mg/d }\end{array}$} & \multirow{3}{*}{$\begin{array}{l}750-2000 \\
\mathrm{mg} / \mathrm{d}\end{array}$} & $<750$ & 1 & 2 & 0 & 0 & 1 & 1 & \multirow{3}{*}{$\begin{array}{l}\mathrm{P}=.117 \\
\mathrm{X} 2=4.287\end{array}$} \\
\hline & & $750-2000$ & 3 & 6 & 9 & 18 & 12 & 12 & \\
\hline & & $>2000$ & 46 & 92 & 41 & 82 & 87 & 87 & \\
\hline \multirow{3}{*}{$\begin{array}{l}\text { Fluid intake } \\
\mathrm{ml} / \mathrm{d}\end{array}$} & \multirow{3}{*}{$\begin{array}{l}500-1000 \\
\mathrm{ml} / \mathrm{d}\end{array}$} & $<500$ & 0 & 0 & 0 & 0 & 0 & 0 & \multirow{3}{*}{$\begin{array}{l}P=.025^{*} \\
X^{2}=5.005\end{array}$} \\
\hline & & $500-1000$ & 2 & 4 & 9 & 18 & 11 & 11 & \\
\hline & & $>1000$ & 48 & 96 & 41 & 82 & 89 & 89 & \\
\hline \multirow{2}{*}{$\begin{array}{l}\text { Potassium } \\
\mathrm{mEq} / \text { IBW/d }\end{array}$} & \multirow{2}{*}{$\begin{array}{l}40 \\
\mathrm{mEq} / \mathrm{IBW} / \mathrm{d}\end{array}$} & $<40$ & 44 & 88 & 38 & 76 & 82 & 82 & \multirow{2}{*}{$\begin{array}{l}\mathrm{P}=.118 \\
\mathrm{X}^{2}=2.439\end{array}$} \\
\hline & & $\geq 40$ & 6 & 12 & 12 & 24 & 18 & 18 & \\
\hline \multirow{3}{*}{$\begin{array}{c}\text { Phosphorus } \\
\text { mg/ IBW/d }\end{array}$} & \multirow{3}{*}{$\begin{array}{l}10-16 \\
\mathrm{mg} / \mathrm{IBW} / \mathrm{d}\end{array}$} & $<10$ & 3 & 6 & 8 & 16 & 11 & 11 & \multirow{3}{*}{$\begin{array}{l}\mathrm{P}=.258 \\
\mathrm{X}^{2}=2.711\end{array}$} \\
\hline & & $10-16$ & 16 & 32 & 16 & 32 & 32 & 32 & \\
\hline & & $\geq 16$ & 31 & 62 & 26 & 52 & 57 & 57 & \\
\hline \multirow{3}{*}{$\begin{array}{l}\text { Calcium } \\
\text { mg/d }\end{array}$} & \multirow{3}{*}{$\begin{array}{l}500-800 \\
\mathrm{mg} / \mathrm{d}\end{array}$} & $<500$ & 15 & 30 & 18 & 36 & 33 & 33 & \multirow{3}{*}{$\begin{array}{l}P=.707 \\
X^{2}=.694\end{array}$} \\
\hline & & $500-800$ & 23 & 46 & 19 & 38 & 42 & 42 & \\
\hline & & $\geq 800$ & 12 & 24 & 13 & 26 & 25 & 25 & \\
\hline
\end{tabular}

$X^{2}$ : Chi-Square test

*significant at $P \leq 0.05, \mathrm{P}=.025 *$

Table (8): dietary intake of some vitamins among studied hemodialysis patients $(\mathrm{n}=100)$

\begin{tabular}{|c|c|c|c|c|c|c|c|c|c|}
\hline \multirow[b]{2}{*}{ Vitamins } & \multirow{2}{*}{$\begin{array}{l}\text { Normal } \\
\text { range }\end{array}$} & \multirow{2}{*}{$\begin{array}{l}\text { Patient } \\
\text { result }\end{array}$} & \multicolumn{2}{|c|}{$\begin{array}{l}\text { Port Said } \\
\mathrm{n}=50\end{array}$} & \multicolumn{2}{|c|}{$\begin{array}{l}\text { Cairo } \\
\mathbf{n}=50\end{array}$} & \multicolumn{2}{|c|}{$\begin{array}{l}\text { Total } \\
\mathrm{n}=100\end{array}$} & \multirow{2}{*}{ Significance } \\
\hline & & & No & $\%$ & No & $\%$ & No & $\%$ & \\
\hline \multirow{3}{*}{ Vitamin B1 (thiamin) } & \multirow{3}{*}{$\begin{array}{l}1.1-1.2 \\
\mathrm{mg} / \mathrm{d}\end{array}$} & $<1.1$ & 49 & 98 & 49 & 98 & 98 & 98 & \multirow{3}{*}{$\begin{array}{l}\mathrm{P}=.753 \\
\mathrm{X}^{2}=.000\end{array}$} \\
\hline & & $1.1-1.2$ & $\overline{1}$ & 2 & 1 & 2 & 2 & 2 & \\
\hline & & $>1.2$ & 0 & 0 & 0 & 0 & 0 & 0 & \\
\hline \multirow{3}{*}{$\begin{array}{l}\text { Vitamin } \\
\text { (riboflavin) }\end{array}$} & \multirow{3}{*}{$\begin{array}{l}1.1-1.3 \\
\mathrm{mg} / \mathrm{d}\end{array}$} & $<1.1$ & 34 & 68 & 39 & 78 & 73 & 73 & \multirow{3}{*}{$\begin{array}{l}\mathrm{P}=.526 \\
\mathrm{X}^{2}=1.285\end{array}$} \\
\hline & & $1.1-1.3$ & 4 & 8 & 3 & 6 & 7 & 7 & \\
\hline & & $>1.3$ & 12 & 24 & 8 & 16 & 20 & 20 & \\
\hline \multirow{3}{*}{ Vitamin C } & \multirow{3}{*}{$\begin{array}{l}75-90 \\
\mathrm{mg} / \mathrm{d}\end{array}$} & $<75$ & 47 & 94 & 48 & 96 & 95 & 95 & \multirow{3}{*}{$\begin{array}{l}P=.366 \\
X^{2}=2.011\end{array}$} \\
\hline & & $75-90$ & 0 & 0 & 1 & 2 & 1 & 1 & \\
\hline & & $>90$ & 3 & 6 & 1 & 2 & 4 & 4 & \\
\hline \multirow{3}{*}{ Vitamin A } & \multirow{3}{*}{$\begin{array}{l}75-90 \\
\mathrm{mg} / \mathrm{d}\end{array}$} & $<75$ & 46 & 92 & 47 & 94 & 93 & 93 & \multirow{3}{*}{$\begin{array}{l}\mathrm{P}=.603 \\
\mathrm{X}^{2}=1.011\end{array}$} \\
\hline & & $75-90$ & 3 & 6 & 3 & 6 & 6 & 6 & \\
\hline & & $>90$ & 1 & 2 & 0 & 0 & 1 & 1 & \\
\hline
\end{tabular}


Table (9): Relation between Socio-demographic characteristics and protein intake among studied hemodialysis patients $(\mathrm{n}=100)$

\begin{tabular}{|c|c|c|c|c|c|c|c|c|c|}
\hline \multirow{4}{*}{\multicolumn{2}{|c|}{ Socio-demographic data }} & \multicolumn{8}{|c|}{ Protein } \\
\hline & & \multicolumn{4}{|c|}{$\begin{array}{c}\begin{array}{c}\text { Port Said } \\
\mathbf{n}=\mathbf{5 0}\end{array} \\
\end{array}$} & \multicolumn{4}{|c|}{$\begin{array}{l}\text { Cairo } \\
\mathbf{n}=50\end{array}$} \\
\hline & & $\begin{array}{l}<1 \\
\text { g/kg/day }\end{array}$ & $\begin{array}{l}1-1.2 \\
\text { g/kg/d } \\
\text { ay } \\
\end{array}$ & $\begin{array}{l}>1.2 \\
\mathrm{~g} / \mathrm{kg} / \mathrm{da} \\
\mathrm{y}\end{array}$ & \multirow[t]{2}{*}{$\begin{array}{l}\text { Significan } \\
\text { ce }\end{array}$} & $\begin{array}{l}<1 \\
\text { g/kg/da } \\
y\end{array}$ & $\begin{array}{l}1-1.2 \\
\text { g/kg/da } \\
y\end{array}$ & $\begin{array}{l}>1.2 \\
\mathrm{~g} / \mathrm{kg} / \mathrm{da} \\
\mathrm{y}\end{array}$ & \multirow[t]{2}{*}{$\begin{array}{l}\text { Significanc } \\
\text { e }\end{array}$} \\
\hline & & No $(\%)$ & $\begin{array}{l}\text { No } \\
(\%)\end{array}$ & No $(\%)$ & & No $(\%)$ & No $(\%)$ & No $(\%)$ & \\
\hline \multirow{4}{*}{ Education } & Illiterate & $0(0)$ & $2(4)$ & $1(2)$ & \multirow{4}{*}{$\begin{array}{l}P=.031 * \\
X 2=13.910\end{array}$} & $4(8)$ & $3(6)$ & $3(6)$ & \multirow{4}{*}{$\begin{array}{l}P=.025^{*} \\
X 2=14.466\end{array}$} \\
\hline & $\begin{array}{l}\text { Read and } \\
\text { write }\end{array}$ & $0(0)$ & $1(2)$ & $3(6)$ & & $4(8)$ & $5(10)$ & $4(8)$ & \\
\hline & $\begin{array}{l}\text { Intermediate } \\
\text { School }\end{array}$ & $7(14)$ & $2(4)$ & $21(42)$ & & $12(24)$ & $1(2)$ & $7(14)$ & \\
\hline & $\begin{array}{l}\text { University } \\
\text { Degree }\end{array}$ & $6(12)$ & $3(6)$ & $4(8)$ & & $0(0)$ & $1(2)$ & $6(12)$ & \\
\hline \multirow{4}{*}{$\begin{array}{l}\text { Marital } \\
\text { Status }\end{array}$} & Single & $2(4)$ & $0(0)$ & $2(4)$ & \multirow{4}{*}{$\begin{array}{l}\mathrm{P}=.151 \\
\mathrm{X} 2=9.430\end{array}$} & $3(6)$ & $0(0)$ & $1(2)$ & \multirow{4}{*}{$\begin{array}{l}P=.013 * \\
X 2=16.105\end{array}$} \\
\hline & Married & $10(20)$ & $5(10)$ & $25(50)$ & & $14(28)$ & 7 (14) & $17(34)$ & \\
\hline & Divorced & $0(0)$ & $1(2)$ & $0(0)$ & & $3(6)$ & $0(0)$ & $2(4)$ & \\
\hline & Widowed & $1(2)$ & $2(4)$ & $2(4)$ & & $0(0)$ & $3(6)$ & $0(0)$ & \\
\hline
\end{tabular}

$X^{2}$ : Chi-Square test

*significant at $P \leq 0.05, P=.031 *, p=.025 *, p=.013 *$.

\section{DISCUSSION:}

The current study aimed to assess nutritional needs for patients undergoing hemodialysis. This aim was achieved by the present study findings and the research questions were answered. This study was found that, HD patients evaluated in this study showed significant shortcoming in terms of quantitative and qualitative measure of food intake, when compared with specific recommendations for individuals in hemodialysis and guidelines for a healthy diet. In addition, there are significances between sociodemographic variables, dietary habits and nutritional needs and must be taken into consideration in the nutritional assessment.

Hemodialysis patients commonly have poor dietary habits, particularly with regard to the intake of foods with high concentrations of sugar and fats, and low levels of consumption of cereals, fruits and vegetables, an observation that is consistent with the findings of this study. Shortcomings in the intake of calories, proteins, saturated fats, cholesterol, vitamins and minerals, among other food components, are also found by other researchers (Sanlier\& Demircioglu 2007; Khoueiry\& Waked 2011; Vaz\& Freitasa et al. 2014), as was the case of the current study results.

According to the results yielded by the current study, the majority of HD patients in both group are taking less than $35 \mathrm{kcal} / \mathrm{kg} / \mathrm{day}$ and more than one- fifth of HD patients are taking less than $1 \mathrm{~g} / \mathrm{kg} / \mathrm{day}$ did not reach the recommended intakes for dietary energy and protein, respectively; so they were at risk of malnutrition and increase mortality rate. A number of studies point to a lack of intake of energy and proteins by Bossola \&Leo (2013) were reported that, the daily intake of energy was also inadequate in the majority of patients with mean dietary calorie intake was $22.17 \pm 5.125 \mathrm{kcal} / \mathrm{kg}$ per day and dietary protein was intake less than $1.2 \mathrm{~g} / \mathrm{kg} / \mathrm{day}$. Also, Hajira \&Samiullah (2013) were observed that, the intake of protein and energy are frequently low because of the underlying disease, psychosocial factors, and 
uremic anorexia. Among the individuals evaluated in this study, average intake of proteins was adequate in more than two- fifth of HD patients. This finding was in consistent with Piccini\& Fairburn (2014) were mentioned that, increased the daily intake of protein was more than or equal $1.0 \mathrm{~g} / \mathrm{kg} / \mathrm{d}$ or greater which increased survival rates than HD patients below this level. In addition, Vaz\&Freitas (2014) who concluded that, the average intake of dietary protein $1.18 \pm 0.23$ grams of proteins per kilogram of adjusted weight. In the point of view of the researcher, HD patients have lack information about nutritional education and healthy knowledge.

In order to improve the consumption of proteins and calories among the patients evaluated, an increase of these two components in the diet will therefore be recommended. To increase protein intake, the consumption of meat with a lower phosphorus/ protein ratio and less fat can be recommended, since these foods provide proteins with high biological values and contribute to improved iron intake, something which was lacking in the patients in this and in other studies of hemodialysis patients (Sanlier\& Demircioglu 2007; Khoueiry\& Waked et al. 2011). This is why a daily portion of meat and eggs is recommended for a healthy diet but is usually insufficient for hemodialysis patients, who need higher levels of protein and at least $50 \%$ of protein with high biological levels (NKF/DOQI, 2000).

In addition, A strategy to improve calorie intake for the individuals evaluated would be to increase the consumption of complex carbohydrate, with an emphasis on cereals and whole foods and a reduction in simple carbohydrates, since nearly half of patients consumed less than the six daily portions of cereals recommended (Brasil, 2006), and had excessive levels of consumption of sugar and sweets. This measure should also be useful to improve the intake of fibers that was close to the minimum recommended levels and would increase consumption of proteins, since whole foods such as bread, rice and others, have reasonable levels of these elements (Tabela\& Composicao, 2011).

The current study was revealed that, the average intake of carbohydrate, fats and cholesterol among studied HD patients in this study was consistent with levels recommended by international and national guideline for hemodialysis patients (NKF-K/DOQI 2000; Fouque \& Vennegoor 2007; Rucker \& Thadhani et al. 2010). With respect to other characteristics of dietary intake, the diet of HD patients was relatively healthy. Bossola $\boldsymbol{\&}$ Leo (2013) were concluded that, the diet of Italian HD patients was relatively healthy: daily carbohydrate intake as a percentage of the total caloric intake was $51.8 \% \pm 8.9 \%$, the daily lipid intake as a percentage of total caloric intake was $32.1 \% \pm 7.1$. In addition, $78 \%$ of patients had a cholesterol intake less than $300 \mathrm{mg} /$ day. In the point view of the researcher, hypercholesterolemia of studied HD patients may be due to their method of cooking related to patient and nursing knowledge deficiency about sources of cholesterol and saturated fat intake and eaten pattern which less than half of the study samples used foundry and sautéed method in cooking.

Although the average intake of fats among HD patients in the current study was consistent with levels recommended for hemodialysis patients, qualitative shortcomings were observed, on account of the increased consumption of saturated fats and cholesterol in (39\%) of patients and an excess of excess of oils and fats. Chronic kidney disease sufferers often have dyslipidaemia and although food intake is not a cause of this disorder, inadequate consumption of lipids can aggravate the 
condition (Lima, 2007). Since the average level of lipid consumption was appropriate, instead of promoting a reduction in intake of this macronutrient, which would result in a lower calorie intake, there would be a greater impact if the quality of fat consumed by the patients under evaluation was changed. Lou \&Campos (2014) were reported that measures such as moderating red and processed meat consumption and prioritizing lean meats along with poultry and fish are relevant strategies for reducing saturated fat and cholesterol intake, while the daily consumption of olive oil contributes to a better balance between polyunsaturated fatty acids and monounsaturated fats.

The current study was revealed that, the mean daily dietary fiber intake was lower than the recommended value of less than $20 \mathrm{~g} / \mathrm{day}$, in almost all patients. Bossola \&Leo (2013) was observed that, patients receiving dialysis consume significantly lower amounts of dietary fiber. In the general population, a higher intake of fiber has been associated with a lower prevalence of important risk factors for CVD, including hypertension, obesity and type 2 diabetes mellitus (Streppel\& Arends, 2005).

The current study was elicited that, there is no statistically significance relation in dietary intake of macronutrients, fiber and malnutrition between both groups. Chen\& Peng (2013) was found that, no significant differences of calories, protein, fat, carbohydrates, dietary fiber, and cholesterol intake were found between the normal nutrition group and malnutrition group $(\mathrm{P}>0.05)$.

With regard to dietary sodium and fluid, the average intake higher the recommended level. The current study was elicited that, there was a statistically significance relation in dietary intake of fluid between both group whereas $(\mathrm{P} \leq 0.05)$. Stark \&Snetselaar (2011) were reported that HD patients consumed higher sodium fast foods and convenience foods which higher in sodium and lead to increase fluid intake. Also, Clark\& Sontrop (2011) was found that, there is a benefit to increase fluid intake to hydrated individuals in CKD patients. However, this finding is in disagreement with Bossola \&Leo (2013) was concluded that HD patients have a low intake of sodium. This low intake is possibly due to the fact that HD patients know that intake of salt increases their thirst, and so they maintain it as low as possible. Also, Hajira (2013); Vaz et al. (2014) who mentioned that the average intake of sodium below the minimum recommended level. In the point view of the researcher, HD patients often rely on higher sodium fast foods, eating pickles and convenience foods, which that increase the thirst and leading to increase in fluid intake and in IDWG. Consequently, education on ways to control thirst and lower the intake of both sodium and fluid is one important nutritional strategy in hemodialysis patients.

The current study was revealed that, the average intake of potassium lower than the recommended level. In the point view of the researcher, given the need to control the intake of potassium, may be due to many hemodialysis patients end up reducing their consumption of fruits, vegetables, and greens. A fact that was also observed in this study which the study HD patients consumed of fruits, vegetables and greens 1-2 T/W. However, although these foods are a significant source of potassium, their consumption should be encouraged in order to ensure a balance between fibers, vitamins and minerals. The choice of fruit, vegetables and greens with lower levels of this mineral, controlling portion sizes and the frequency of consumption of potassium rich foods and the avoidance of boiling certain vegetables (Cuppari \& Amâncio, 2004), will ensure an intake in compliance with the recommended. 
For the individuals evaluated, increased consumption of fruit, vegetables and greens will improve the intake of fibers, retinol and Vitamin C. However, more than one fifth of the study samples have hyperkalemia. Severe hyperkalemia can cause fatal arrhythmias, HD patients should receive guidance detailed about the potassium content of various food and should be instructed to avoid or restrict the ingestion of food rich in potassium as potato, banana, orange juice, dried fruits, nuts, tomato sauce, beans, peas, chocolate, among others (Riella, 2001).

With regard to dietary phosphorus, the average intake was higher than the recommended level. Concerning dietary data, (dietary record and food frequency table) in this study showed that the dietary phosphorus content is directly related to the quantity and type of protein, legumes such as beans foul medames, tamiaa, processed meats and dairy products, especially cheese milk, in the diet and carbonated drinks. Although the average consumption of milk and dairy products was found to be lower than the levels recommended for a healthy diet (Brasil,2006), increased ingestion of these foods to the recommended three daily portions would not be advised, since it would imply an increased supply of phosphorus in the diet. In the point view of the researcher, more than half of the HD patients have hyperphosphatemia due to the majority of food in this groups have a relationship between phosphorus and protein that is higher (Carvalho \& Cuppari, 2011). Control of hyperphosphatemia is vital to the long-term well-being of renal failure. Dietary restriction of phosphorus intake and adequate dialysis therapy form the basis for management, but are usually insufficient to bring about adequate control. Since the phosphorus intake is closely related to the protein intake, and since dialysis is relatively inefficient in phosphorus removal, most patients on replacement therapy require the use of phosphate binding agents to reduce the intestinal absorption of dietary phosphorus. In the point view of the researcher, more than half of the HD patients have hyperphosphatemia may be due to deficit knowledge for patient and nursing about protein sources which contain lower phosphorus.

Concerning dietary calcium the average of intake within the recommended level in more than two fifth of HD patients. Moreover, more than one fifth HD patients the average of intake lower than the recommended level. Lou\&Campos (2007) ; Cupisti \&Alessandro et al. (2010) were reported that, the average intake of calcium below the recommended level. Concerning dietary data, (dietary record and food frequency table) in this study showed that the average consumption of milk and dairy products was found to be lower than the levels recommended for a healthy diet. The low levels of consumption of these foods might have contributed towards the increased prevalence of inadequate levels of retinol and may explain the reduced average intake of calcium that was found, something which should not give rise to concern, since part of the calcium that comes from some of the creations of phosphorus is absorbed and should be considered as a non-dietary source of this mineral (Fouque \& Vennegoor 2007; Carvalho \& Cuppari et al. 2011).

In the point view of the researcher, the phosphorus restriction makes the diet unpalatable, especially if the protein intake is high. A diet rich in protein and calcium and poor in phosphorus is difficult to obtain a careful exclusion of some foods and meticulously monitoring phosphate plasma level, will allow the use of lowest possible amount of phosphate-binding products. Calcium salts are effective phosphate binders provided that they are taken during meals. Calcium carbonate or acetate supplements are largely used for intestinal binding of phosphate, to correct metabolic acidosis and 
to provide the required calcium supplements. In relation to sociodemographic data, the current study was found that, there was a statistical significance between sociodemographic variables, dietary habits and nutritional needs and must be taken into consideration in the nutritional assessment.

\section{CONCLUSION:}

The patients evaluated in this study showed significant shortcoming in terms of quantitative and qualitative measure of food intake, when compared with specific recommendations for individuals in hemodialysis and guidelines for a healthy diet.

The analysis of dietary patterns showed that, the patients were found to have an imbalanced diet, characterized by an excess of consumption of oils and fats, particularly saturated fats and cholesterol, sugars and sweets, higher sodium and phosphorus intake along with a low level of consumption of iron, fruits, complex carbohydrates ,vegetable oils, vegetables and greens, milk and derivatives and a considerable proportion of patients who did not attain the minimum recommended amounts of intake for calories, protein, retinol, iron and calcium. Correcting these problems within each case possibilities would allow for a reduction in saturated fat consumption and higher sodium, phosphorus intake, balancing the diet with foods from our geographical area; so dietetic counseling and education is very important in HD patients, as well as reevaluation of type of dietary protein and energy requirements.

\section{RECOMMENDATION:}

Based on the findings of the present study, the following recommendations were suggested:

1- Regular assessment of nutritional needs and status of patients undergoing maintenance hemodialysis every three to six months, to identify patient's at malnutrition risk, and allow for early nutritional intervention.

2- Education and dietary counseling should be the first step in attempting to maintain adequate energy and protein intake. If this approach is unsuccessful, nutritional support such as supplementing formula, tube feeding, or Intradialytic and parenteral nutrition is recommended.

3- Monitor serial body weight and body mass index aiming to maintain the high normal body mass index, and to be concerned about progressive loss of weight even in overweight patient.

4- Restriction of dietary phosphorus intake with a combination of phosphorus binders, in case of high protein needs, hyperphosphatemia, hypocalcemia and higher BUN, is recommended.

5- Restriction of dietary sodium intake which influence on increase fluid intake, IDWG and hypertension in the study patients, in case of hypernatremia and increase IDWG, is recommended and restriction of saturated fat and cholesterol, in case of hypercholesterolemia.

6- The importance of multidisciplinary collaborative working between doctors, nurses and specialist dietitians to detect patients that encounter a nutritional problem. 


\section{Further studies:}

1- It is recommended conducting this study on large sample with different geographical characteristics.

2- These data come from dialysis patients in stable condition and free from severe comorbidities, so the results are not exactly representative of the overall HD population.

3- Further research should assessment for nutritional needs of hemodialysis patients in Egypt which it is a little.

4- Further research should investigate for the important role of the diet in improvement HD patient's conditions.

5- It is advised to conduct experimental studies using dietary counseling and dietary education programs for HD patients, which promote HD patients dietary intakes of food.

6- It is advised to conduct experimental studies in dietary training programs for the medical staff about nutritional guidelines of dietary intakes.

\section{Acknowledgements}

Researchers would like to thank all the staff in the critical care units in the selected hospitals and all patients who participated in this study.

\section{REFERENCES:}

Afifi, A., Moustafa, R., El Setouhy, M., El Sayed, E., Ghafar, S., \& Karim, M. (2008): The Egyptian Renal Registry, 9th Annual Report, Prevalence of ESRD in Egypt study.Pp.8-9.

Amira, C., Bello, B., \& Braimoh, R. (2015): Chronic kidney disease: a ten-year study of a etiology and epidemiological trends in Lagos, Nigeria. British journal of medicine; 19(4).Pp.19:23.

Bossola, M., Leo, A., Viola, A., Carlomagno, G., Viola, A., Monteburini, T., Cenerelli, S., Santarelli, S., Boggi, R., Miggiano, G., Vulpio, C., Mele, C., \& Tazza, L. (2013): Dietary intake of macronutrients and fiber in Mediterranean patients on chronic hemodialysis. JNEPHROL; doi: 10.5301/jn.5000222. 26(5): Pp.912-918.

Brasil. M.D.S (2006): General Coordination of Food and Nutrition Policy. Food Guide for the Brazilian population: promoting healthy eating. Brasilia: Ministry of Health.

Burrows, H. (2005): Chronic kidney disease: an overview. American Journal of Nursing. 105(2):Pp.40-49.

Cahyaningsih, N. (2009): Indonesian Nephrology Nurse Association (PPGII). The role of dialysis nurse, Community Nursing Improvement, nerscomite; 30(6). Available at: http://b11nk. wordpress.com/2009/08/24/the-role-of-dialysisnurse/.Accessed in 28/12/2014. 
Carvalho, A., \& Cuppari, L. (2011):: Control of hyperphosphatemia. DRC.J Bras Nefrol. doi: 10.1590/S0101-28002011000200012. 33(1):Pp.1-6.

Centers for Disease Control and Prevention, (2010): National Chronic Kidney Disease Fact Sheet: General Information and National Estimates on Chronic Kidney Disease in the United States. Atlanta, GA: U.S. Department of Health and Human Services, CDC. Available at: http://www.cdc.gov/diabetes/pubs/factsheets/ kidney.htm.Accessed in 25/12/2012.

Chen, J., Peng, H., Xiao, L., Zhang, K., Yuan, Z., Chen, J., Wang, Z., Wang, J., \& Huang, H. (2013): Inflammation but not dietary macronutrients insufficiency associated with the Malnutrition-Inflammation Score in hemodialysis population. .doi:10.1371/journal.pone.0083233. 8 (12): e83233.

Clark, W., Sontrop, J., Macnab, J., Suri, R., Moist, L., Salvadori, M., \& Garg, A. (2011): Urine Volume and Change in Estimated GFR in a Community-Based Cohort Study. American Society of Nephrology. doi:10.2215/CJN.01990211. 6 (11): Pp.2558-2560.

Cupisti, A., D'Alessandro, C., Valeri, A., Capitanini, A., Meola, M., Betti, G., \& Barsotti, G. (2010): Food intake and nutritional status in stable hemodialysis patients. doi: 10.3109/ 08860220903391234. 32(1): Pp. 47-54.

Cuppari, L., Amâncio, O., Nobrega, M.,\&Sabbaga, E. (2004): Vegetable preparation for diet arestrita potassio use. Nutrire; 28(5):Pp.17-30.

De Mutsert, R., Grootendorst, D., Indemans F., Boeschoten, E., Krediet, R., \& Dekker, F. (2009): Association between serum albumin and mortality in dialysis patients are partly explained by inflammation, and not by Malnutrition. Journal of Renal Nutrition Mar. 19(2):Pp.127-135.

De Oliveira, G., Andrade, E., Acurcio, F., Cherchiglia, M., \& Correia, M. (2012): Nutritional assessment of patients undergoing hemodialysis at dialysis centers in Belo Horizonte, MG, Brazil. Rev. Assoc. Med. Bras. São Paulo. doi. http://dx.doi.org/10.1590/S0104-42302012000200022. 58 (2):Pp.240-247.

Fouque, D., Vennegoor, M., Wee, P., Wanner, C., Basci, A., Canaud, B., Haage, P., Konner, K., Kooman, J., Martin-Malo, A., Pedrini, L., Pizzarelli, F., Tattersall, J., Tordoir, J., \& Raymond, V. (2007): EBPG guideline on nutrition. Nephrol Dial Transplant; doi: 10.1093/ndt/gfm020. 22(2):Pp.45-87.

Hajira, B., Samiullah, M., \& Chawla, K. (2013): Nutritional Status Assessment of Hemodialysis Patients. ARPN Journal of Agricultural and Biological Science. 8(4), ISSN 1990-6145. Available at: www.arpnjournals.com. Accessed in 15/04/2014.

Khoueiry, G., Waked, A., \& Goldman, M. (2011): Dietary intake in hemodialysis patients does not reflect a heart healthy diet. J Ren Nutr ; 21(6):Pp.438-447.

Lima, J. (2007): Dyslipidemia in chronic kidney disease. Rev Soc Cardiol State São Paulo; 17(1):Pp.60-65. 
Macafee, S., Magee, D., \& Laughlin, H. (2011): Evidence based dietetic guidelines protein requirements of adults on haemodialysis and peritoneal dialysis BDA renal nutrition group, the British Dietetic Association, RNG Haemodialysis Group. Pp. 4.

National Kidney Disease Education Program, (2014): Improving the understanding, detection, and management of kidney disease, Dialysis, U.S. Department of health and human services. Available at http://www.nkdep.nih.gov/living/kidneyfailure/dialysis.shtml. Accessed in 25/12/2014.

National Kidney Foundation, (2000): Clinical practice guidelines for nutrition in chronic renal failure. K/DOQI, National Kidney Foundation. American Journal of Kidney Diseases 35, Pp. S1-140.

Poulia, K., and Baschali, A.(2014): European Dialysis and Transplant Nurses Association/ European Renal Care Association EDTNA/ERCA. Detecting and Treating Malnutrition in People with Chronic Kidney Disease.

Ribeiro, M., Araújo, M., Netto, M., \& Cunha, L. (2011):Dining habits of the impact on the dietary profile of hemodialysis patients. J Bras Nefrol. doi: 10.1590/S0101-28002011000100010. 33(1):Pp.69-77.

Rucker, D., Thadhani, R., \& Tonelli, M. (2010): Trace element status in hemodialysis patients, Seminars in Dialysis, 23(4):Pp.389-395.

Sanlier, N., and Demircioglu, Y. (2007): Correlation of dietary intakes and biochemical determinants of nutrition in hemodialysis patients. Ren Fail. doi: 10.1080/08860220601098904.29(2):Pp.213-218.

Smeltzer, S., Bare, B., Hinkle, J., \& Cheever, K. (2012): Brunner \&Suddarth's textbook of medical-surgical nursing. - 12th .ed. Lippincott Williams \& Wilkins. Philadelphia, London.

Stark, S., Snetselaar, L., Hall, B., Stone, R., Kim, S., Piraino, B., \& Sevick, M. (2011): Nutritional Intake in Adult Hemodialysis Patients, Top ClinNutr, 26 (1), Pp. $45-56$.

Streppel, M., Arends, L., Veer, P., Grobbee, D., \& Geleijnse, J.(2005): Dietary fiber and blood pressure: a meta-analysis of randomized placebo-controlled trials. Arch Intern Med; 165 (2): Pp.150-156.

Tabela, B., and Composicao, D.(2011):Campinas : Unicamp ; 4th .ed. Disponívelem: Available at: http://www.unicamp.br/ nepa/taco/tabela.php. Accessed in 25/03/2015.

Thomas, N., and Coldstream, F. (2006): Managing chronic kidney disease. In the community British Journal of Renal Medicine.11 (1):Pp.26-28.

Vaz, I., Freitasa, T., Peixotom, D., Ferraz, S., \& Campos, M. (2014):Food intake in patients on hemodialysis. Rev.Nutr.,Campinas, ,nov./dez. doi.org/10. 1590/141552732014000600002. 27(6):Pp.665-675. 
تقييم الآحتياجات الغذائية للمرضى الخاضعين للغسيل الكلوى

دينا جمال حسين ، أ .د/ماجدة عبد العزبز محد ،د/ أبمان صالح شاهبن ، د/أمل بكر أبو العطا

\section{الخلاصة}

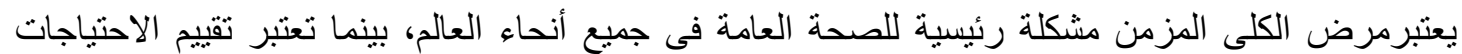

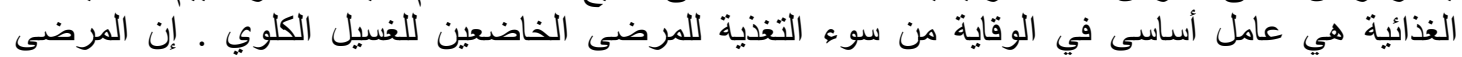

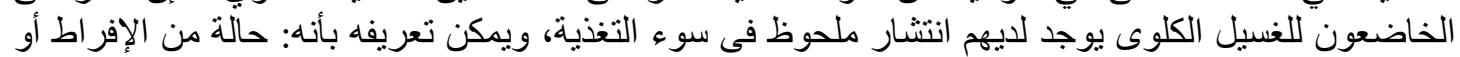

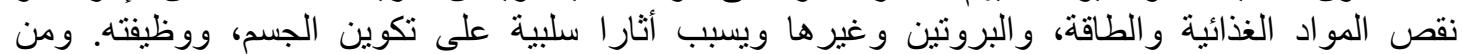

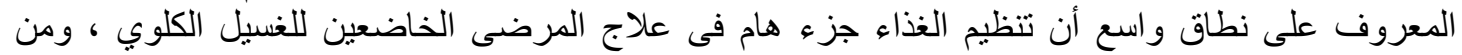

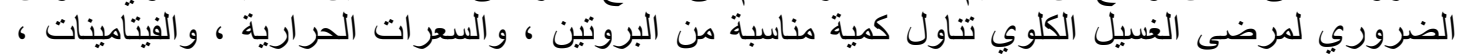

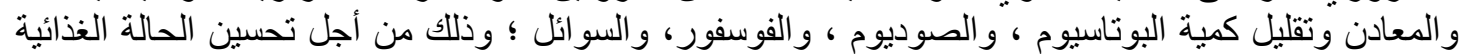

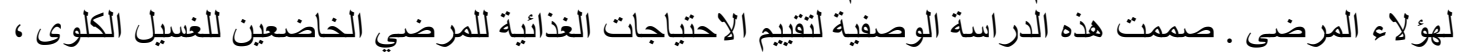
ومنثم الخروج بالتوصيات الغذائية الملائمة .

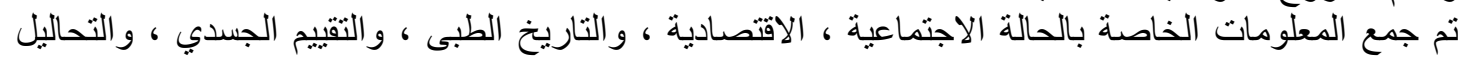

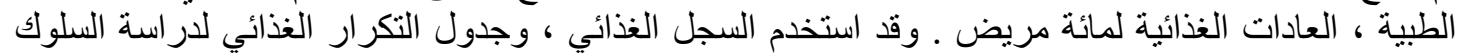

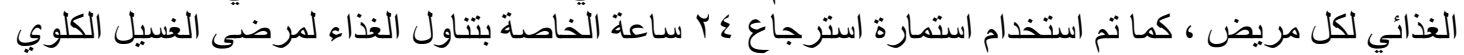

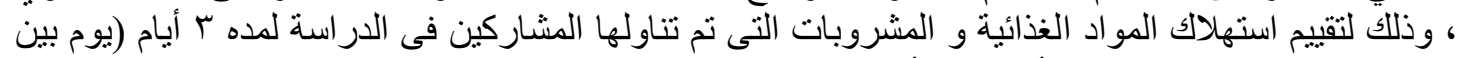

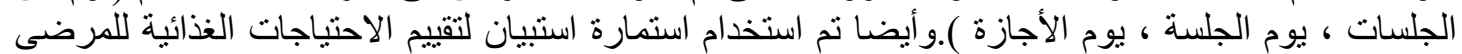

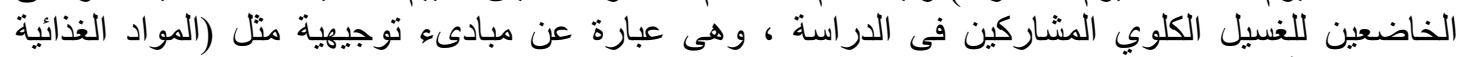

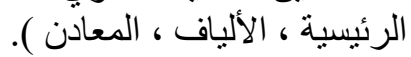

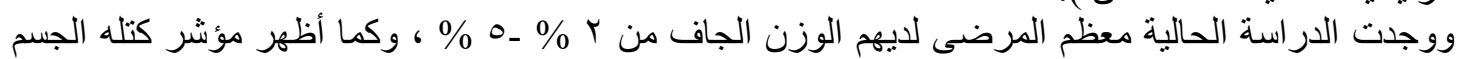
لاى أكثر من نصف المرضهى من (BMI)

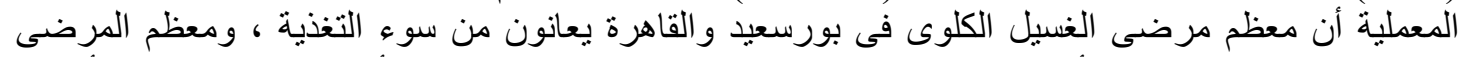

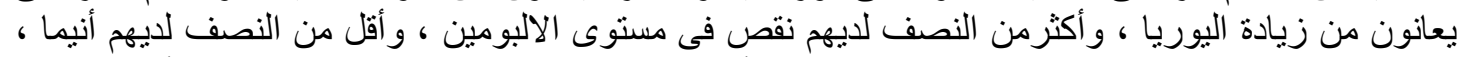

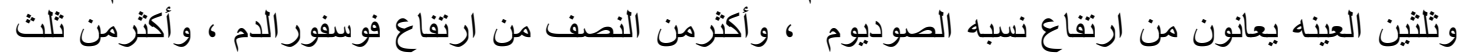

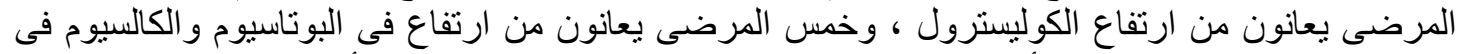

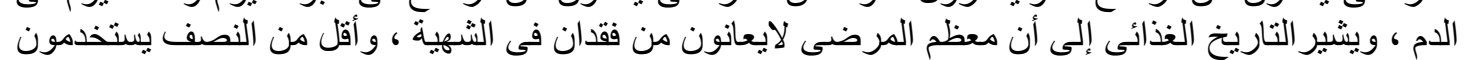

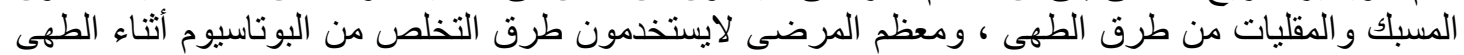

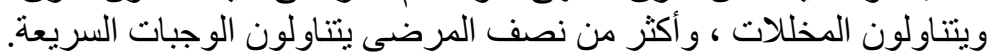

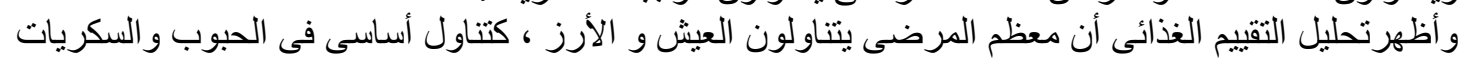

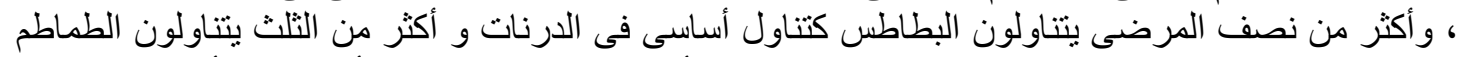

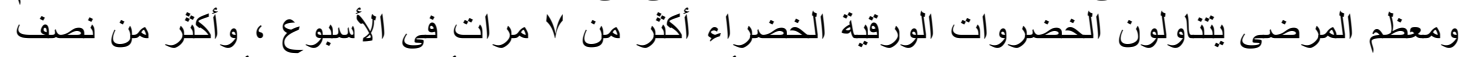

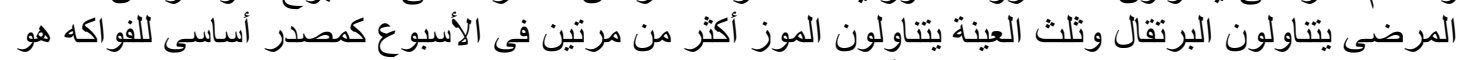

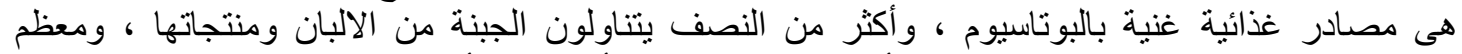

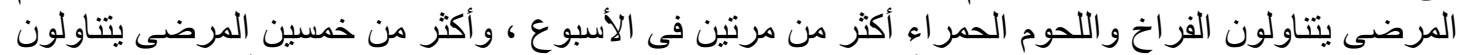

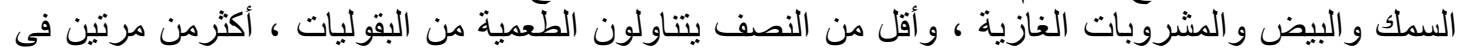

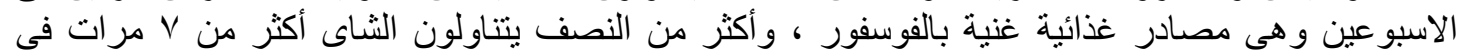

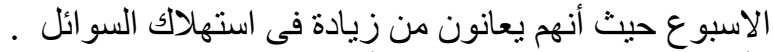

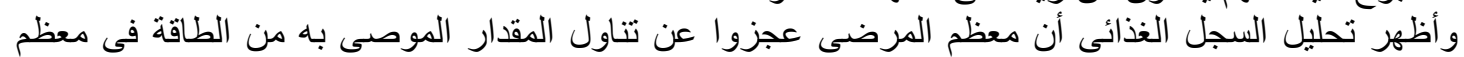

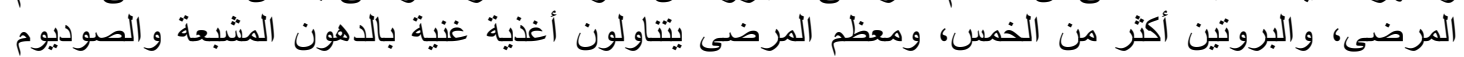

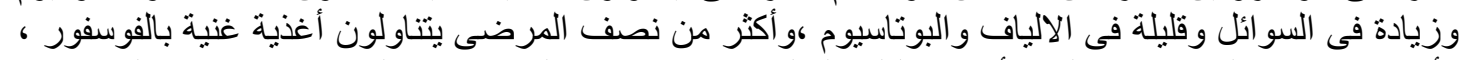

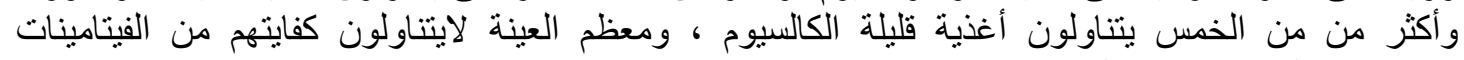

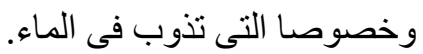

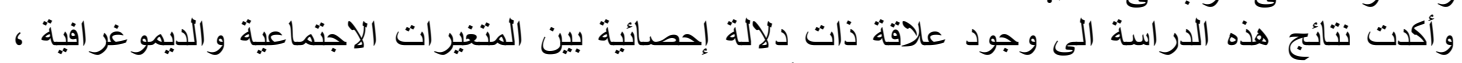

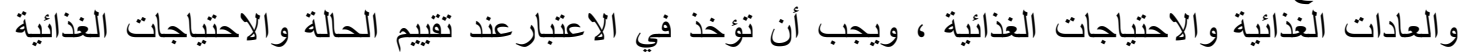
للمرضى الخاضعين للغسيل الكلوى . 